\title{
Diffusion in the asteroid belt
}

\author{
Harry Varvoglis \\ Section of Astrophysics, Astronomy and Mechanics, Department of Physics, University of \\ Thessaloniki, GR-541 24 Thessaloniki, Greece \\ email: varvogli@physics.auth.gr
}

\begin{abstract}
In the beginning we review briefly the evolution of the ideas on the motion of the bodies in our solar system, from Newton's clockwork Universe to the presently accepted ubiquity of chaotic transport in the asteroid belt. Then we discuss the result of chaotic motion, which is transport in phase space, and we introduce the concept of diffusion of an asteroid in action space. We proceed by reviewing recent work on numerical as well as analytical study of asteroids following chaotic trajectories and we summarize the main results. We present several applications of the theoretical modelling of asteroid motion as diffusion in action space, to problems of specific interest.
\end{abstract}

Keywords. Asteroid belt, chaos, diffusion

\section{Introduction}

Celestial Mechanics, the first branch of Astronomy where mathematical modelling managed to interpret and predict celestial phenomena, has a record of impressive achievements. In less than three hundred years, starting from Newton's law of gravitation and his three laws of dynamics, Celestial Mechanics managed finally to accurately describe, in practice, the trajectories of all planets and minor bodies of our solar system. The theory, behind all this, was based on the implicit assumption that the functions describing all these trajectories had all the "nice" mathematical properties and, in particular, that they were analytic in all variables and parameters, including the initial conditions. Astronomers were aware, of course, since the work of Poincaré, that the simplest model used in Celestial Mechanics to describe perturbed motion, the planar circular restricted three-body problem, does not posses any other analytic integrals of motion, besides the Jacobi integral. The general consensus was, however, that the homoclinic and heteroclinc tangles, described so vividly by Poincaré, were of little importance to the actual problem of solar system dynamics, at least for time scales of practical interest.

Things started to change in the last half of the twentieth century, after the seminal work by Hénon and Heiles (1965) revealed that chaos in the homoclinic tangle, as described by Poincaré, may dominate the phase space of very simple dynamical systems. Soon, through the work of Giffen (1973) in the 2:1 and Scholl \& Froeschlé (1974) in the 3:1 resonance, it was understood that the simplest model used in Celestial Mechanics, the planar restricted three-body problem, possesses non-ignorable chaotic regions in phase space. Subsequently Wisdom (1980) showed that chaos in the outer belt is due to the overlap of first-order resonances and derived the law

$$
\Delta a=1.5 a_{J} \mu^{2 / 7}
$$

which, in the model of the circular three-body problem, relates the width, $\Delta a$, of the resonance-overlap region around the orbit of the secondary body, to its mass, $\mu$, and semimajor axis, $a_{J}$. Later it was shown that a similar effect is produced by the overlapping of the components of high-order resonant multiplets (Murray \& Holman (1997)) in the 
model of the elliptic three-body problem. In this way it became widely accepted that chaos is an important phenomenon in the solar system. Therefore Newton's idea of a clockwork universe, which, once started to move, would continue moving in the same way "ad perpetuum", suddenly was proved to be wrong. The bodies of the solar system, especially the minor ones, may follow trajectories that change secularly in time, so that "collisions" (either true or just close encounters) and ejections play an important role as sinks (both) and sources (the former) of bodies, even in the present era. As a result, the question now is not anymore whether chaos affects the dynamics of planetary systems, but on which time-scales it does so and on whether it is the rule or the exception. Recent calculations put the percentage of main belt asteroids on chaotic orbits at $30 \%$ (proper elements and LCE computations, Milani \& Knežević (2003)).

However it was not so easy to understand how to treat mathematically the dynamical evolution of bodies on chaotic trajectories, since this was a novel situation in Celestial Mechanics. Laplace's theory and its continuation, proper elements theory, are not valid in chaotic regions. As numerical experiments clearly show, the values of proper elements in chaotic regions change in a secular, non-quasi-periodic, way. This is how the concepts and methods of Statistical Physics, in particular transport theory, were introduced in Celestial Mechanics. In the rest of this article we will review these methods and show how transport theory may be used in order to obtain useful information in real problems of Celestial Mechanics.

\section{Transport vs. diffusion}

The "classical" way to treat a system, in which certain quantities change in an erratic way with time, is the statistical approach. Statistical Physics has developed a wide range of tools that may be used in the description of such a system. Basic concept in this respect is the concept of diffusion. Here, however, one should be very careful, since the term diffusion has been sometimes used in a liberal way in Celestial Mechanics. In Statistical Physics the term diffusion has a restrictive meaning. Speaking in simple terms, it describes the evolution of an ensemble of $n$ "particles", undergoing a classical random walk in some state variable(s), i.e. a process described by a random variable with finite second moments. Even more restrictive is the set of available tools for the mathematical description of such a system. They consist, essentially, in a set of partial differential equations, named transport equations. Principal among these equations is the Fokker-Planck equation (e.g. see Lichtenberg \& Lieberman (1992))

$$
\frac{\partial f}{\partial t}=-\frac{\partial(B f)}{\partial x}+\frac{\partial^{2}(D f)}{\partial x^{2}}
$$

which gives the evolution of the probability density function, $f(x, t)$, of an ensemble of "particles" that undergo a random walk in $x$-space. $\dagger$ The first term in Eq. (2.1) describes drift, i.e. a systematic motion, through the frictional coefficient $B$, and the second diffusion, i.e. a random motion, through the diffusion coefficient $D$, in the one-dimensional $x$-space. The equation can be easily generalized for more than one degrees of freedom.

In the case of conservative dynamical systems, the drift term can be incorporated into the diffusion term, due to the conservation of phase space volume, so that in the simplest possible case of a 1-D conservative system the Fokker-Planck equation takes the simple

$\dagger$ I.e. $f(x, t) d t$ gives the probability that, at time $t$, the co-ordinate $x$ of a "particle" lies within the interval $x$ and $x+d x$. 
form,

$$
\frac{\partial P}{\partial t}=\frac{\partial}{\partial I}\left(\frac{D}{2} \frac{\partial P}{\partial I}\right)
$$

in which enters only one parameter, the diffusion coefficient $D(I) . \dagger$ Note that in Eq. (2.2) we have used action-angle variables, $I-\theta$, and this is not accidental, since this selection has two important consequences. First in the case of a perturbed integrable system, where the action is an integral of the integrable part, any "random" change in the action may be considered, under some conditions which will be discussed later, as a diffusion. The second is that the "randomness" of the process can be easily put in mathematical terms, by invoking the random phase approximation, in which we assume that the angle variable is evolving in a random way. The above mathematical formulation seems to fit very well to the problems encountered in Celestial Mechanics, where the overwhelming majority of the models used are conservative perturbations of the Keplerian motion and they are very often cast in Hamiltonian form and action-angle variables (Delauné, Poincaré, or any modified form). However, there is a small, but crucial, point, that makes the application of the Fokker-Planck equation to problems of Celestial Mechanics less than straightforward. This point is that, due to the degeneracy of the Hamiltonian describing planetary motion in general, the osculating elements of a regular orbit in the three-body problem are not constant, even in the integrable (Laplace) approximation, but undergo secular oscillations. Therefore one has to separate the "regular" change of the elements, due to secular motion, from the "diffusive" one, due to the chaotic nature of the motion. This was pointed out by Murray \& Holman (1997) and can, in principle, be accounted for by using in Eq. (2.2) free elements, instead of osculating. However in practice this imposes a lot of supplementary computational work, which makes cumbersome the numerical solution of the problem.

In the derivation of the Fokker-Planck equation several assumptions are implicit, so that, before using it to describe transport in a real problem, one has to ensure that all these assumptions really hold. Otherwise there is a danger that one could end up by solving correctly a wrong model! In particular, one should check the following conditions.

- First we should confirm that the set of trajectories, whose evolution we are trying to calculate, lie in a chaotic region of phase space. The chaotic nature of the region should by confirmed by calculating the Lyapunov number(s) (LCNs).

- The second condition is that the chaotic region, in action space, should be simply connected.

- The third condition is that the motion could be considered as a Markovian process, which means that there should be a fast decay of correlations.

- The fourth condition is that the "time-step" of the random walk model should be larger than the autocorrelation time, $\Delta t \gg t_{c}$, while the "typical" change in action should be small, $\Delta I \ll I$.

- The fifth condition is that the variance of the random-walk probability density function should be finite, i.e. $\sigma \neq \infty$.

Now it is instructive to give some examples of cases of interest in Celestial Mechanics, which cannot be described mathematically by a Fokker-Planck equation, at least in a straightforward way.

- Real dynamical systems posses a divided phase space, i.e. the chaotic regions are closely intermingled with ordered ones. If the measure of the ordered regions is an

$\dagger$ The diffusion coefficient might depend on time as well, i.e. $D=D(I, t)$, but the discussion of this case lies outside the scope of the present article. 


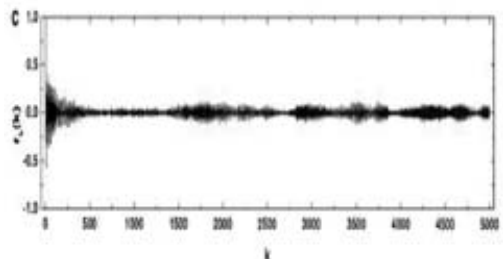

I

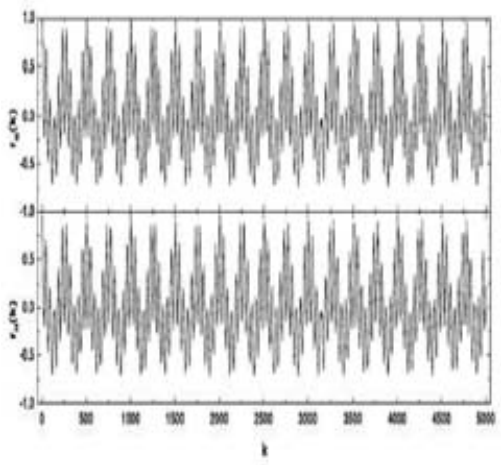

Figure 1. The autocorrelation functions of the three Delaunay variables of a stable chaotic asteroid. The one corresponding to the semi-major axis drops almost exponentially, an indication that the variations of the semi-major axis are chaotic. The autocorrelation functions of the other two actions, however, oscillate, roughly, between, +1 and -1 , an indication that the variation of $G$ and $H$ are quasi-periodic, so that they cannot be modelled as diffusion in the corresponding action space. Taken from Tsiganis, Varvoglis \& Hadjidemetriou (2002a).

important fraction of the total available phase space, the simple statistical description (either theoretical or numerical) fails.

- When the second condition is not satisfied, a few "holes" in phase space may be accounted for by introducing absorbing or reflecting barriers. But a complicated selfsimilar topology, like the one existing at the border between ordered and chaotic regions, might lead to Lévy-like statistics (see fifth item below).

- The third condition excludes several transport processes that appear in chaotic systems. E.g. in systems with more than one degrees of freedom some of the degrees might be strongly chaotic, so that the maximal Lyapunov exponent has a large value, while others might be so mildly chaotic, as to appear ordered on physically important time-scales. In this way the particles diffuse, in the space of the strongly chaotic degrees, on a much shorter time-scale than in the space on the mildly chaotic degrees. A typical example is the case of stable chaos in the solar system (Milani \& Nobili (1992)). In a stable chaotic trajectory, the autocorrelation function of one action (corresponding to the semi-major axis) decays on a time scale of the order of the Lyapunov time (i.e. the inverse of the maximal Lyapunov number). However, the autocorrelation functions of the other two actions (corresponding to the eccentricity and the inclination) vary quasi-periodically for thousands of Lyapunov times (Fig. 1). Therefore correlations in the eccentricity do not decay fast and the process cannot be considered as diffusion in eccentricity and/or inclination space (although it might be considered as diffusion in semi-major axis space).

- The variation of the "normal", non-resonant action variables in the vicinity of a low-order resonance cannot be considered as diffusion, since it fails to fulfill the third and the fourth conditions, hence the need to use "free" elements.

- Finally the fifth condition is a subtle one. The cornerstone of classical Statistical Mechanics is the Central Limit Theorem, which holds if the variance of a random variable 
is finite. If this is true for the random variable related to the random walk of the diffusing particles, the process is classical diffusion and one arrives naturally from the FokkerPlanck equation, Eq. (2.2), to Fick's law

$$
\sigma^{2}(t)=\left\langle[I(t)-\langle I(t)\rangle]^{2}\right\rangle=D t
$$

where the average is taken over all particles and over the total time interval. If this is not true, then

$$
\sigma^{2}(t) \propto t^{b}
$$

with $b \neq 1$. This is exactly the regime of the non-classical Lévy diffusion (Metzler \& Klafter (2000)), named after the French mathematician who first described it. In particular the dependance of the variance might be either faster than linear in time $(b>1$, super-diffusion) or slower ( $b<1$, sub-diffusion). An interesting example of super-diffusive behavior in Celestial Mechanics has been found recently by Carruba et al. (2003), who showed that close encounters with Ceres disperse the asteroids semi-major axes with a power-law exponent $b>1$, i.e. faster than Fick's law.

It has been postulated that, at least some cases of slow chaos in the asteroid belt, are manifestations of sub-diffusion. This situation appears, in particular, in the regions of a dynamical system close to the border dividing order from chaos. Presently there is no widely accepted mathematical description of this type of transport. Several authors $†$ have proposed transport equations with fractional derivatives, which might account not only for a Markovian process with infinite second moments, but for processes with memory as well. However these equations are very hard, in general, to solve, either analytically or numerically. The present practice is to model these processes by a Fokker-Planck equation, whose diffusion coefficient is selected ad hoc, so that its solutions mimic a Lévy probability density function.

\section{Statistical approach and Numerical Experiments}

The first authors to attempt a statistical description of chaotic motions in the asteroid belt was the group of Lecar (e.g. Lecar, Franklin \& Murison (1992), Lecar, Franklin \& Soper (1992), Murison, Lecar \& Franklin (1994)). They performed numerical experiments, by integrating the equations of motion for a large number of asteroids and they calculated the Lyapunov time of each trajectory as well as a "characteristic" time, which was meant to describe the time needed for the eccentricity of an asteroid to reach a planet crossing value, after which it would soon be ejected from the main asteroid belt due to a close encounter. They arrived at a very surprising conclusion: the plot of the "characteristic" times vs. the Lyapunov times showed a linear trend in a log-log plot. They interpreted this result as a "universal law" in the asteroid belt. As it turned out, the trend is real but the correlation coefficient is low, so that it cannot be considered as a law. Lecar and his co-workers found a strong correlation, only because they assigned to Jupiter a mass ten times larger than its real one. But in this way they changed the dynamics of the system, as it was pointed out by Murray \& Holman (1997). In particular, from eq. (1.1) it is evident that in their model all mean motion resonances overlapped, creating a simply connected chaotic region. However this unexpected result triggered a number of papers by other authors, who tried to understand and interpret it. To my opinion this fact was in the heart of the development of the statistical description of chaotic asteroid motion, in general, and of the diffusive approach, in particular.

$\dagger$ e.g. see Metzler \& Klafter (2000) and references therein 
Morbidelli \& Froeschlé (1996) arrived at the conclusion that in the resonance overlap regime there is, indeed, a polynomial dependence between the escape and the Lapunov times, but there is no universal value of the exponent. Shevchenko (1998), working in the modulated pendulum approximation, assumed that the diffusion is taking place near the border of a separatrix and arrived at a the conclusion that such a relation exists and it is, moreover, quadratic. Varvoglis \& Anastasiadis (1996) introduced to this problem a purely statistical approach. They assumed that the asteroid motion is a Markovian process and they wrote down a modified Fokker-Planck equation. Then they attempted to solve the equation in the limiting case of strong perturbation, when the surviving islands of stability are of negligible measure. Assuming, moreover, a diffusion coefficient depending only on the value of the Lyapunov number and not on the action, they solved the equation analytically and showed that a relation similar to the one found by the Lecar group is naturally recovered. These authors suggested as well that the motion of Helga, the first example of stable chaos found by Milani \& Nobili (1992), could not be modelled as diffusion in eccentricity space.

\section{Diffusion of asteroids - Mathematical formulation}

In the framework of the three-body problem, asteroids can follow chaotic trajectories when in the vicinity of a mean motion resonance. Due to the ellipticity of Jupiter's orbit, each resonance is in fact a multiplet of close-by harmonics. Their overlapping is the main source of chaos.

Two different approaches have been presented so far, with the aim to (i) understand the cause of chaotic diffusion and (ii) provide analytic estimates for the relevant quantities, i.e. the diffusion coefficient and the escape time of chaotic orbits. The Hamiltonian describing the motion of an asteroid in a mean motion resonance of order $q$ has the form (in the 2-D elliptic three-body problem)

$$
\mathcal{H}=\frac{1}{2} \alpha \Psi^{2}+\beta \Phi+\varepsilon \sum_{p=0}^{q} c_{p} \cos (\psi-p \phi)
$$

where the $(\psi, \Psi)$ degree of freedom describes the libration inside the resonance and the $(\phi, \Phi)$ degree of freedom describes the motion of the free pericenter. The last term of the Hamiltonian is the resonant multiplet and $c_{p} \sim \Phi^{p / 2} \sim e^{p}$, where $e$ is the free eccentricity of the asteroid. The harmonics are separated by the amount $\delta \Psi=\beta / \alpha$, while their width is a function of the eccentricity, given by $\Delta \Psi=\sqrt{2 \varepsilon c_{p} / \alpha}$. What controls the dynamics in the resonance is the ratio of the distance between the harmonics, with respect to their widths, $K=\Delta \Psi / \delta \Psi$.

The results of Murray \& Holman (1997) support that $K=\Delta \Psi / \delta \Psi \sim 1$ in most resonances in the outer asteroid belt and, hence, the situation is close to the one shown in Fig. 2(b). In this case we can consider that the variable $\Psi$ performs repeated uncorrelated random jumps of order $\Delta \Psi$ within a time equal to the Lyapunov time. The diffusion in $\Psi$ is bounded within a region of size $\sum \Delta \Psi(\Phi)$, which depends on the eccentricity. At the same time it results to a diffusion in $\Phi \sim e^{2}$, with a diffusion coefficient of the form

$$
D \sim \varepsilon \Phi^{p} \sim \varepsilon c_{p}^{2}
$$

Since, in the resonances where $K=\Delta \Psi / \delta \Psi \sim 1$ it turns out that $c_{p} \sim \varepsilon$, the value of the diffusion coefficient turns out to be of order $\varepsilon^{3}$. Murray \& Holman (1997) used this result to solve the Fokker-Planck equation and derive analytic estimates for the 
a)

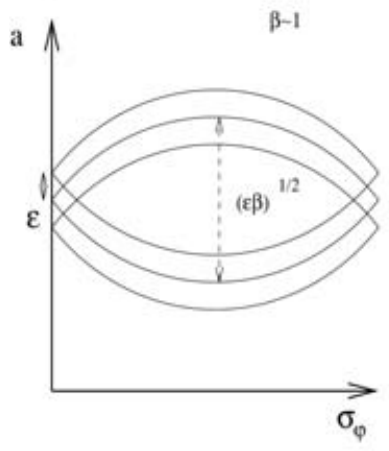

b)

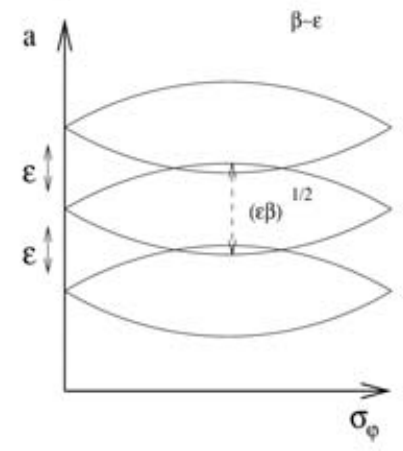

c)

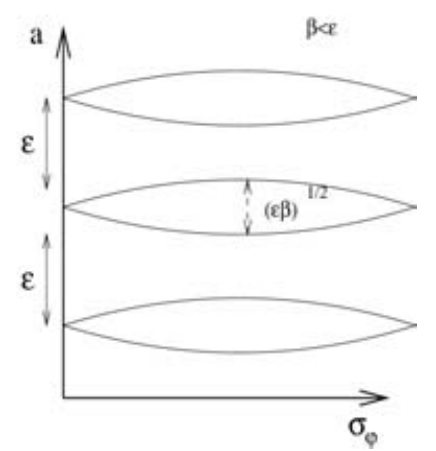

Figure 2. The three different possible arrangements of secondary resonances. Either they completely overlap, in which case the dynamics resemble to that of a modulated pendulum (a), or they overlap partially, in which case the dynamics are those described by Murray \& Holman (1997) (b) or they do not overlap at all, in which case there is no diffusion (c). Taken from Morbidelli \& Guzzo (1996).

escape time of asteroids. Their estimates agree, in general, with numerical integrations conducted in the frame of more advanced models. Although the applicability of this theory in most resonances of the asteroid belt can be questioned, it is an elegant theory providing meaningful results.

The results of Wisdom (1985) for the 3:1 resonance and those of Neishtadt (1987) focus on the situation $K \gg 1$ described in Fig. 2 (a). We note that this approximation holds in low- and medium- order resonances and can take into account important secular effects that appear inside mean motion resonances, as e.g. is the 3:1 case. In fact this approximation, although more laborious than the one presented above, may be more suitable for most of the resonances in the asteroid belt.

When $K \gg 1$, the resonant dynamics resemble those of a slowly modulated pendulum. The variable $\Psi$ is not an appropriate action, as it cannot be considered as having repeated uncorrelated jumps of order $\Delta \Psi$ within a Lyapunov time. The relevant action, which is random walking in the case, is the area of libration of the pendulum, which suffers small changes when the separatrix of the pendulum sweeps by. Neishtadt (1987) has calculated the change in this adiabatic invariant over a secular cycle (i.e. $t \sim 1 / \varepsilon$ ), finding $\Delta \mathcal{J} \sim \varepsilon$. This gives a diffusion coefficient for $\mathcal{J}$ which is of order $D \sim \frac{\langle\Delta \mathcal{J}\rangle^{2}}{\langle\Delta t\rangle} \sim \varepsilon^{3}$. Thus, we find that the diffusive time-scale for resonances of this type is as long as the one estimated through the Murray \& Holman theory, provided (as we discuss in the next paragraph) that the resonance does not support a periodic orbit in the simplest non-trivial approximation of the elliptic planar three-body problem. This result agrees with what numerical integrations show, i.e. test-particles may take more than 1 Gyr to leave a high-order resonance (Tsiganis, Varvoglis \& Hadjidemetriou (2002b), Tsiganis \& Morbidelli (2003)).

However, if the secular dynamics are such that low-eccentricity regions are smoothly connected to high-eccentricity regions, the situation is different. This is the case for e.g. the $3: 1$ resonance, which possesses, in the the planar elliptic restricted problem, a resonant periodic orbit of order 1 . The separatrix of the pendulum intersects the homoclinic orbit and slow diffusion of $\mathcal{J}$ allows the two eccentricity regions to communicate. In this way the eccentricity suffers large intermittent jumps, while $\mathcal{J}$ is slowly diffusing and changes only by small amounts. Using his calculations for $\Delta \mathcal{J}$, Neishtadt (1987) estimated the 


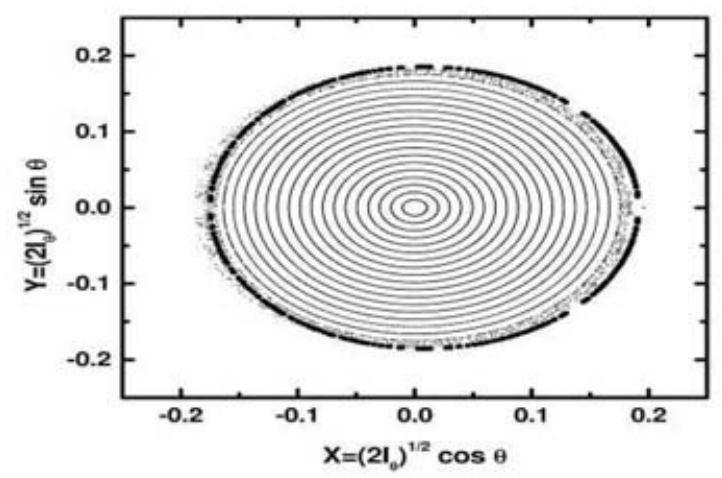

Figure 3. The secular dynamics, in the case of complete overlap, for the resonance $8 / 3$, where there is no periodic orbit. The thick curve, superimposed on the graph, denotes the points of intersection of the $(X, Y)$ plane with the separatrix of the modulated pendulum. All orbits intersecting this curve become chaotic. Notice the small width of the chaotic strip. Taken from Tsiganis \& Morbidelli (2003).

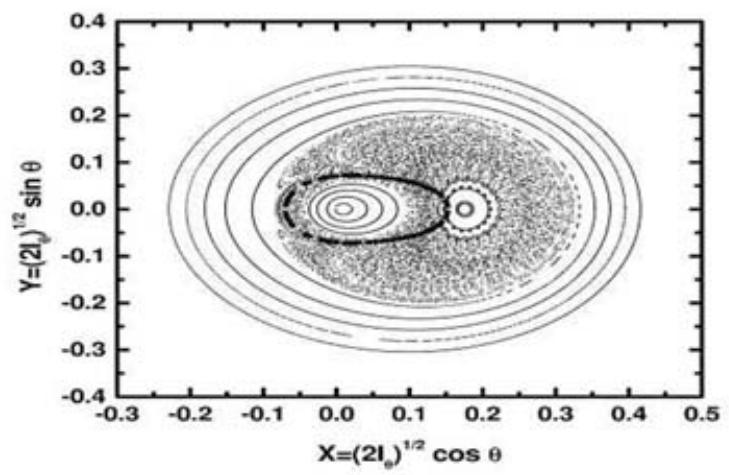

Figure 4. Same as Fig. 3, except that the resonance portrayed here is the $3 / 1$, where there exists a periodic orbit. Notice the extended width of the chaotic region. Taken from Tsiganis \& Morbidelli (2003).

time scale between two eccentricity jumps to be of order $10^{5} \mathrm{yrs}$, as was found earlier by the numerical integrations of Wisdom (1985). This process is the cause of fast, nondiffusive transport in the asteroid belt.

We note that the regime of applicability of the two different approximations for diffusive transport has not been clarified yet. Although a unique theory would be more preferable, it seems that a case-by-case study should be made for mean motion resonances in the asteroid belt.

\section{Applications to specific astronomical problems}

Besides the work on the development of the basic ideas and tools, the statistical approach to problems that involve diffusive motions of asteroids has found already several applications. 


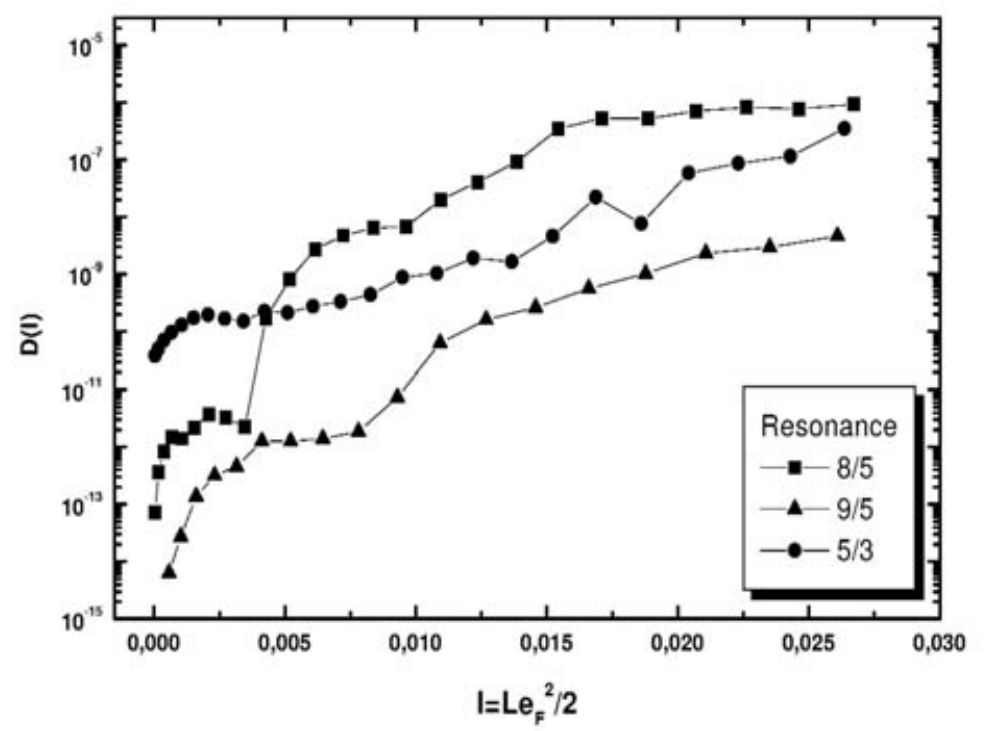

Figure 5. The diffusion coefficient calculated numerically for three different resonances, as a function of the eccentricity of the diffusing body. Taken from Tsiganis, Varvoglis \& Anastasiadis (2003).

\subsection{Numerical calculation of $D(I)$}

Tsiganis, Varvoglis \& Anastasiadis (2003) calculated numerically (Fig. 5) the functions $D(I)$ for the $5 / 3,8 / 5$ and $9 / 5$ resonances in the framework of the elliptic restricted three-body problem. They found that the diffusion coefficient indicates differences not only between low and high eccentricity values, but also between resonances that support a periodic orbit in this model and those that do not.

- (a) The $8 / 5$ and $9 / 5$ resonances, which do not support a periodic orbit, have distinctively different functional behavior at low and high eccentricity regions. In the low eccentricity region they have a "stair-like" form, which is predicted by the theory of Murray \& Holman (1997) and indicates that the dynamics are those of partial resonance overlap. The stair-like form is due to the fact that, for different values of $e_{f}$, the strongest term of the resonant multiplet, which determines the exponent $p$ in Eq. (4.2), is different. The small-eccentricity part of these curves can, indeed, be fitted by curves of the form $D(I) \sim I^{b}$. However for $e_{f} \gtrsim 0.20$ this is not possible, because at intermediate eccentricities the dynamics are those of a modulated pendulum.

- (b) The functional form of the diffusion coefficient for the case of the $5 / 3$ resonance cannot be fitted by a power law at all, because in this resonance there exists a periodic orbit in the framework of the elliptical planar restricted three-body problem, the dynamics are similar to those of the $3 / 1$ resonance, and the theory of Neishtadt (1987) should be more appropriate.

Finally, at high eccentricities (say, $e_{f} \gtrsim 0.25$ ) all curves converge to the same limit, $D=D_{Q L}$, the quasi-linear value, which is approached as all mean motion resonances begin to overlap.

\subsection{Chronology of the Veritas family}

It has been found (Milani \& Farinella (1994)) that several members of the Veritas family, including the largest body in the family, asteroid (490) Veritas itself, are located inside 


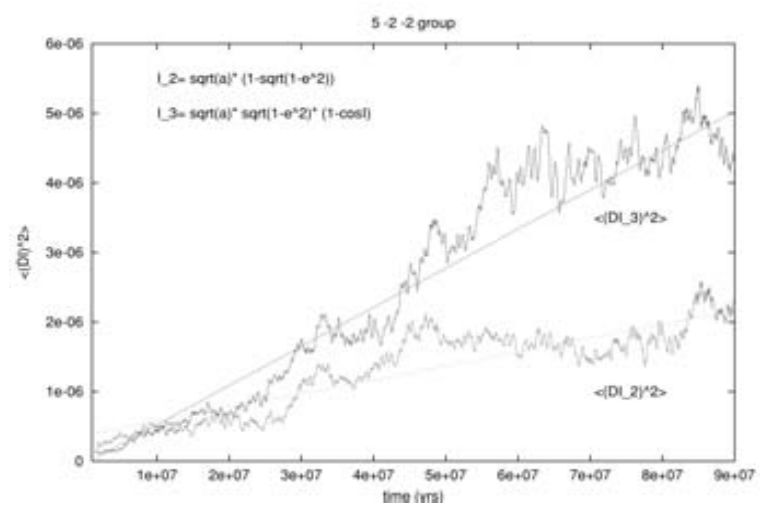

Figure 6. Morbidelli \& Nesvorný (1998) have shown that some members of the Veritas family are in the $(5-2-2)$ resonance. The evolution of the variance of the $I_{2}$ and $I_{3}$ actions, as a function of time, for the chaotic members of this group is linear in time. The action $I_{2}$ is related to the eccentricity and the action $I_{3}$ is related to the inclination. Note the different value of the diffusion coefficient in the two actions. Taken from Tsiganis, Knežević \& Varvoglis (2004).

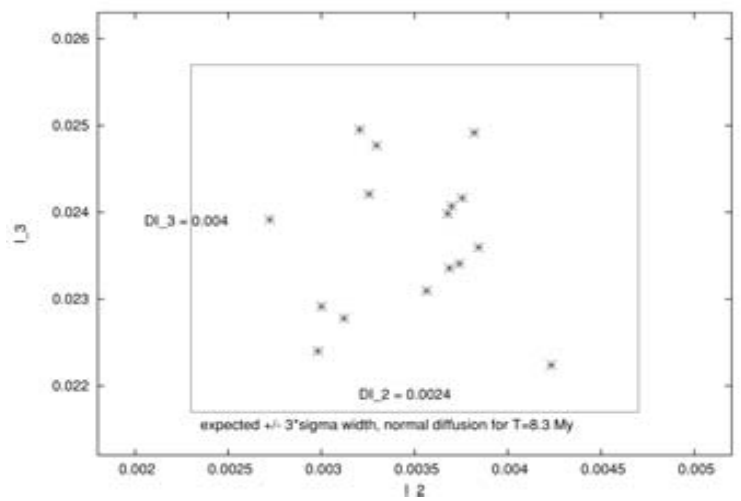

Figure 7. The chaotic members of the Veritas family in the $(5-2-2)$ resonance plotted on the $I_{2}-I_{3}$ space. The frame of the figure corresponds to $3 \sigma(t)$, for $t=8.3 \mathrm{Myrs}$, in both variables. From the fact that all asteroids in this group lie within the frame, we can infer that the age of the family is $\approx 8.3$ Myrs. Taken from Tsiganis, Knežević \& Varvoglis (2004).

the chaotic zone and exhibit a typical chaotic behavior. Lyapunov times for these bodies are of the order of 10,000 yrs and they all appear to be in the resonance overlap regime. Chaotic diffusion brings these bodies outside the family boundaries on a 100 Myrs timescale, which can be interpreted as an upper bound to the age of the family (Milani \& Farinella (1994)). Knežević et al. (2002) confirmed that the motion of the chaotic family members is indeed diffusive, by showing that the evolution of the variance of the eccentricity and inclination obeys Fick's law and, from the slope of the curve $\sigma^{2}(t)=D t$, estimated the diffusion coefficient (Fig. 6). Then, by assuming that the initial probability density function was a $\delta$-function, they compared the analytical solution of the FokkerPlanck equation to the observed distribution of the actual chaotic members of the family. From this they estimated (Tsiganis, Knežević \& Varvoglis (2004)) that the age of the family is $\approx 8.3$ Myrs (Fig. 7 ), a value that agrees nicely with the result found recently by Nesvorný et al. (2003), by back integrating the members of the family on ordered orbits. 


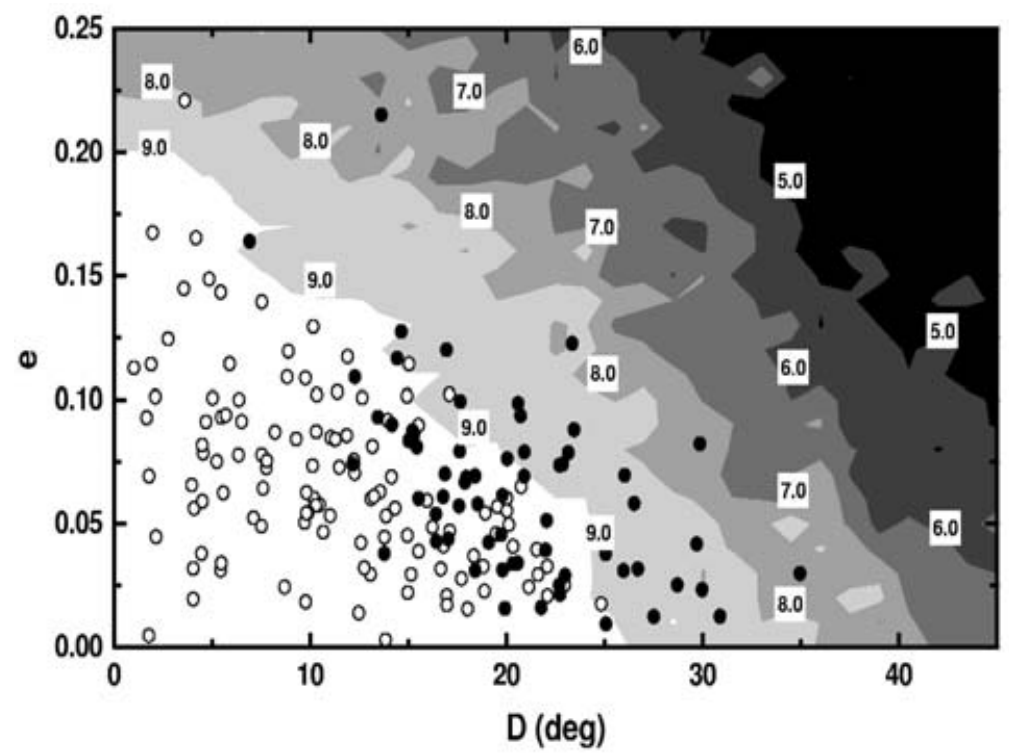

Figure 8. The location of ordered and chaotic real Trojans in the eccentricity (e)-libration width $(D)$ space. The real bodies (white dots ordered, black dots chaotic) are superimposed on the map of the logarithm of escape time (in yrs), derived by integrating fictitious bodies. Taken from Tsiganis, Varvoglis \& Dvorak (2004).

\subsection{Chaotic diffusion of Jupiter Trojans}

We now know that many Jupiter Trojans follow chaotic orbits (Milani (1993)). Many types of secondary resonances exist inside the 1:1 libration region (see Robutel et al. 2004), resulting in a slow chaotic motion, which resembles a diffusive evolution of the width of libration. Levison et al. (1997) had already shown, by means of numerical experiments, that the Trojans are slowly dispersing with time. However, the question whether this slow depletion is the result of chaotic diffusion or of a non-conservative phenomenon (e.g. collisions or Yarkovsky) remained open for some years.

Tsiganis, Varvoglis \& Dvorak (2004) extended the numerical results of Levison et al. (1997). They derived the distribution of regular and chaotic bodies in the Trojan swarms, as a function of the proper elements. They also defined the "effective stability region", in which a Trojan has an escape time greater than the age of the solar system (see Fig. 8). For objects escaping within 1 Gyr, they showed that a power-law trend, relating the Lyapunov and escape time scales, exists (Fig. 9). As the boundary of stability is approached, this power-law trend is severely distorted, a fact that indicates a non-classical diffusive behavior. Finally, using the observations they estimated the diameters of the Trojans and derived the distributions of diameter for (i) the population of ordered and (ii) the population of chaotic Trojans. As shown in Fig. 10, the two distributions were found to be nearly identical, which shows that the transport of Trojans from the outskirts of the effective stability region towards the chaotic domain is a size-independent process, i.e. chaotic diffusion instead of radiation forces or collisions.

\section{Discussion and conclusions}

Proper elements theory is considered as the ultimate tool for studying the long time evolution of the Solar System, in general, and the asteroid belt, in particular. It is 


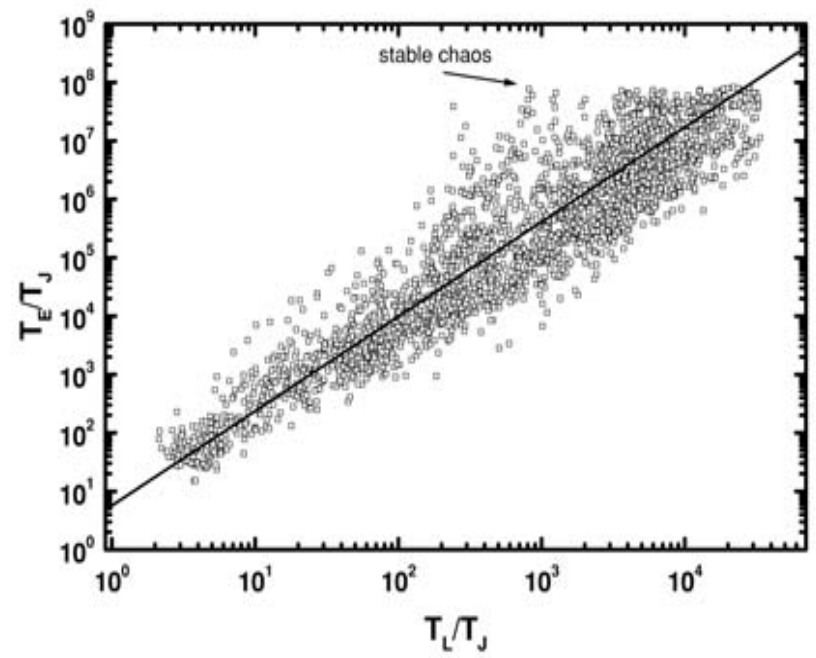

Figure 9. The relation between the Lyapunov time of Trojans and their escape time (through a collision with Jupiter). Several cases of stable chaos are noted. Taken from Tsiganis, Varvoglis \& Dvorak (2004).

interesting to note that proper elements turn out to be of use not only in the case of asteroids on ordered motion, when the corresponding theory is valid, but even in some cases of mildly chaotic trajectories. However the theory has its limitations and becomes meaningless for asteroids with Lyapunov times less than $10^{4}$ years. For these cases it might be more appropriate to change the way of approach and, instead of trying to make accurate predictions for the motion of individual bodies, it could be more instructive to seek the statistical evolution of a whole ensemble. According to the theory of LaplaceLagrange, the semi-major axis does not show important secular changes, so that we focus our attention to the evolution of eccentricity and inclination. If the process in the corresponding actions can be considered as classical diffusion, the appropriate mathematical tool that can help us in this task is the Fokker-Planck equation, which in the case of conservative systems contains only one parameter, the diffusion coefficient. Therefore all information that can be drawn from the point of view of Statistical Physics depends only on the knowledge of the diffusion coefficient and the initial distribution of the ensemble of asteroids.

However even the functional form of the diffusion coefficient is not the same throughout the asteroid belt. Presently there exist two different theories for this form, both for the simplest possible model of the planar elliptic three-body problem, each one applying to a different topological structure of the chaotic region near an orbital resonance. If the various harmonics of the resonant multiplet overlap partially, a situation that holds usually in high-order resonances, the theory by Murray \& Holman (1997) assumes that the action corresponding to the eccentricity is diffusing, with a diffusion coefficient of the form $D \propto \varepsilon c_{p}^{2}$, where $p$ is the order of the harmonic. If the harmonics of the resonant multiplet are located almost on top of each other, a situation that appears usually in low- and medium-order resonances, the theory of Neishtadt (1987) assumes that the action diffusing is the one corresponding to the adiabatic invariant $\mathcal{J}$, with a diffusion coefficient that is of order $\varepsilon^{3}$, where $\varepsilon \sim 10^{-3}$ is the mass of Jupiter (in solar masses). In the cases where low-order resonant periodic orbits in the ERTBP or the 3DRTBP do 


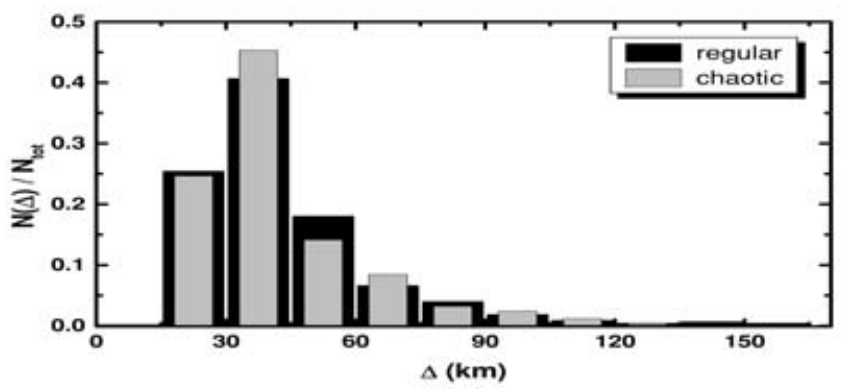

Figure 10. The distribution of regular and chaotic Trojans, with respect to their diameters. Taken from Tsiganis, Varvoglis \& Dvorak (2004).

not dominate the dynamics, the diffusion described by the two theories gives the correct diffusive time-scale, although the physical processes are totally different. Of course in the real world the topology should be, as a rule, an intermediate case between the two limiting ones, something that complicates the theoretical treatment of any real problem.

Things become even more complicated by the fact that the above theories do not address all the possible simple cases. If there exists a resonant periodic orbit in the framework of the model used to describe the dynamical evolution of the ensemble, i.e. the ERTBP, something that in general is true for resonances of low order, transport in eccentricity space is not anymore purely diffusive and it cannot be described solely by any diffusion coefficient. Besides this case, there exists another phenomenon that complicates the statistical study of asteroid evolution, stable chaos. Stable chaotic trajectories are characterized by autocorrelation times that vary by orders of magnitude between each degree of freedom, the autocorrelation time of the semi-major axis being usually the shortest. In this way the evolution of the eccentricity cannot be considered as a random walk and the diffusive approach fails completely. Therefore the diffusive approach has to be applied with caution on a case by case base.

Despite all the above complications, the diffusive approach and its tools turned out to be of use in attacking several real problems. In the present article we presented three such applications. In the first, the numerical calculation of the diffusion coefficient is used in order to differentiate between the two mathematically developed models of diffusion in the specific cases of orbital resonances. In the second, the age of the Veritas family is estimated by assuming a constant diffusion coefficient, which was calculated numerically. In the third case it is concluded that the evolution of Jupiter Trojans is diffusive, on two indications: first that their Lyapunov times have a positive correlation to their escape times, a fact that characterizes diffusion in the border between order and chaos, and second that the distributions of both ordered and chaotic Trojans with respect to their diameters are effectively identical, a fact that precludes the importance of nongravitational forces. It is hoped that in the near future the theoretical understanding of diffusion in the asteroid belt will enable the tackling of more sophisticated problems.

\section{Acknowledgements}

Part of this article is based on work that has been done in collaboration with Profs. R. Dvorak and J. Hadjidemetriou and Drs. A. Anastasiadis, A. Morbidelli and 
K. Tsiganis. I would like to thank Drs. A. Morbidelli and K. Tsiganis, who read a first draft of the article and made many useful comments.

\section{References}

Carruba, V., Burns, J.A., Bottke, W. \& Nesvorný, D. 2003, Icarus 162, 308

Giffen, R., 1973, Astron. Astrophys. 23, 387

Holman, M. \& Murray, N. 1996, Astron. J. 112, 1278

Knežević, Z., Tsiganis, K. \& Varvoglis, H. 2002, in: B. Warmbein (ed.), Asteroids, Comets, Meteors - ACM2002 (ESA SP-500), p. 335

Lecar, M., Franklin, F. \& Murison, M. 1992, Astron. J. 104, 1230

Lecar, M., Franklin, F. \& Soper, P. 1992, Icarus 96, 234

Levison, H., Shoemaker, E.M. \& Shoemaker, C.S. 1997, Nature 385, 42

Lichtenberg, A.J. \& Lieberman, M.A. 1992, Regular and Chaotic Dynamics, (New York: Springer)

Metzler, R. \& Klafter, J. 2000 Phys. Rep. 339, 1

Milani, A. 1993 Cel. Mech. Dyn. Astron. 57, 59

Milani, A. \& Knežević, Z. 2003, http://hamilton.dm.unipi.it/cgi-bin/astdys/astibo

Milani, A. \& Farinella, P. 1994 Nature 370, 40

Milani, A. \& Nobili, A. 1992, Nature 357, 569

Morbidelli, A. 2002, Reg. Chaotic Dyn. 111, 1718

Morbidelli, A. \& Froeschlé, C. 1996, Cel. Mech. Dyn. Astron. 63, 227

Morbidelli, A. \& Guzzo, M. 1996, Cel. Mech. Dyn. Astron. 65, 107

Morbidelli, A. \& Nesvorný, D. 1998, Astron. J. 116, 3029

Murison, M.A., Lecar, M. \& Franklin, F.A. 1994, Astron. J. 108, 2323

Murray, N. \& Holman, M. 1997, Astron. J. 114, 1246

Neishtadt, A. 1987 J. Appl. Math. Mech. 51, 586

Nesvorný, D., Bottke, W.F., Levison, H.F. \& Dones, L. 2003, Astrophys. J. 591, 486

Robutel, P., Gabern, F. \& Jorba, A. 2004, Cel. Mech. Dyn. Astron., in press

Scholl, H. \& Froeschlé, C. 1974, Astron. Astrophys. 33, 455

Shevchenko, I.I. 1998, Phys. Lett. A 241, 53

Tsiganis, K., Knežević, Z. \& Varvoglis, H. 2004, in preparation

Tsiganis, J., \& Morbidelli, A. 2003, Ann. MCFA 3, 999

Tsiganis, K., Varvoglis, H. \& Anastasiadis 2003, in: A. Celletti, S. Ferraz-Mello \& J. Henrard (eds.), Modern Celestial Mechanics: from Theory to Applications, (Dordrecht: Kluwer), p. 451

Tsiganis, K., Varvoglis, H. \& Dvorak, R. 2004, Cel. Mech. Dyn. Astron. in press

Tsiganis, K., Varvoglis, H. \& Hadjidemetriou, J. 2000, Icarus 146, 240

Tsiganis, K., Varvoglis, H. \& Hadjidemetriou, J. 2002, Icarus 155, 454

Tsiganis, K., Varvoglis, H. \& Hadjidemetriou, J. 2002, Icarus 159, 284

Tsiganis, K., Varvoglis, H. \& Morbidelli, A. 2003, Icarus 166, 131

Varvoglis, H. \& Anastasiadis, A. 1996, Astron. J. 111, 1718

Wisdom, J. 1980, Astron. J. 85, 1122

Wisdom, J. 1985, Icarus 63, 272 